\title{
Chemical expansion affected oxygen vacancy stability in different oxide structures from first principles calculations
}

\author{
Dilpuneet S. Aidhy, ${ }^{*}$ Bin Liu, ${ }^{1}$ Yanwen Zhang ${ }^{1,2}$ and William J. Weber ${ }^{2,1}$ \\ ${ }^{1}$ Materials Science and Technology Division, Oak Ridge National Laboratory, \\ Oak Ridge, TN 37831 \\ ${ }^{2}$ Materials Science and Engineering, University of Tennessee, Knoxville, TN \\ 37996
}

*Corresponding author

1 Bethel Valley

PO Box 2008, MS 6138

Oak Ridge TN 37831

Phone: 8652412720

Email: aidhyds@ornl.gov

\section{Bin Liu}

liub2@ornl.gov

Yanwen Zhang

zhangy1@ornl.gov

William Weber

weberwj@ornl.gov 


\begin{abstract}
We study the chemical expansion for neutral and charged oxygen vacancies in fluorite, rocksalt, perovskite and pyrochlores materials using first principles calculations. We show that the neutral oxygen vacancy leads to lattice expansion whereas the charged vacancy leads to lattice contraction. In addition, we show that there is a window of strain within which an oxygen vacancy is stable; beyond that range, the vacancy can become unstable. Using $\mathrm{CeO}_{2} \mid \mathrm{ZrO}_{2}$ interface structure as an example, we show that the concentration of oxygen vacancies can be manipulated via strain, and the vacancies can be preferentially stabilized. These results could serve as guiding principles in predicting oxygen vacancy stability in strained systems and in the design of vacancy stabilized materials.
\end{abstract}

\title{
Keywords:
}

strain, oxygen vacancies, density functional theory, chemical expansion, interfaces 


\section{Introduction}

Oxygen vacancies have emerged as one of the key ingredients in a variety of materials' phenomenon both of fundamental and technological interests. Besides acting as charge carriers in the conventional ion-transport electrochemical processes ${ }^{1,2}$ such as in oxygen sensors, electrodes and cathodes for solid oxide fuel cells, ${ }^{3}$ and in memristive devices, ${ }^{4,5}$ they have found growing appreciation in the emerging field of transition-metal oxides responsible for magnetic, orbital, electronic and transport properties. ${ }^{6,7}$ There are also reports of high-temperature superconductivity induced by ordered oxygen vacancies. ${ }^{8}$ In addition, the oxygen vacancies are found to act as healing agents in materials under radiation environments, thus paving ways for the design of better radiation tolerant materials for nuclear applications and radioactive waste storage. ${ }^{9}$

In almost all of these fields, tailoring of interfaces in materials is being actively pursued as a design parameter to quantitatively enhance the properties. ${ }^{10}$ For example, in the case of ion-transport, there are now multiple examples from both experiments and computation that show enhanced oxygen conductivity in interfacial materials as compared to bulk materials. ${ }^{11}$ Similarly, recent experimental results have demonstrated that interfaces act as strong sinks for radiation-induced point defects, thus increasing a material's radiation tolerance. ${ }^{12,}{ }^{13}$ Multiferroicity and two-dimensional electron gas (2DEGs) heterostructures are other recent examples where materials' interfacing is being heavily exploited. ${ }^{14,15}$

Oxygen vacancies are common to all these interfacial oxide materials and are either inherently present during synthesis or deposition, or are extrinsically created by adding dopants. Interfacial materials and materials that are used under extreme conditions 
are often strained. The strain in interfacial materials is due to lattice mismatch between two materials, while the strain in bulk materials could be due to temperature or chemical gradients, such as in nuclear reactor environments. Under such conditions, the behavior of oxygen vacancies, both kinetics and thermodynamics, is far from that in the bulk. In pursuit of gaining precise control over oxygen vacancies such that strain could be used as a ' $k n o b$ ' to preferentially functionalize vacancies, advancing the understanding of straininfluenced properties of oxygen vacancies is currently one of the most important areas of research in the oxide community. ${ }^{16}$

Substantial progress has been made in recent years on the strain-induced kinetic properties of oxygen vacancies triggered by a study that revealed eight orders of magnitude increase in oxygen conductivity originating at the interface in $\mathrm{YSZ}_{\mid} \mathrm{SrTiO}_{3}{ }^{17}$ Such high conductivity has yet to be reproduced by other groups; however, from other studies since then, an underlying theme has satisfactorily emerged that oxygen migration barriers can be controlled by interfacial strain, i.e., tensile strain lowers oxygen migration barrier due to stretching of the bonds. ${ }^{11,} 18$ In contrast, the strain-influenced thermodynamic properties of oxygen vacancies are less understood. In a series of papers on non-stoichiometric ceria, the concept of chemical expansion, i.e., expansion or contraction of the lattice dimensions due to the presence of oxygen vacancies has been explored using both experiments and computation. ${ }^{19-22}$ It was shown that oxygen vacancies lead to lattice expansion, and hence increase in lattice volume. A similar study on perovskite $\mathrm{CaMnO}_{3}$ using density functional theory (DFT) calculations also showed that oxygen vacancy formation energy decreases under tensile strain conditions compared to equilibrium bulk, i.e., volume expansion induced by tensile strain stabilizes oxygen 
vacancies. ${ }^{23}$ To develop more in-depth understanding, a much wider exploration across various oxide structure types is required to establish the thermodynamic stability of oxygen vacancies under strained conditions. In an effort to explore this concept in chemically and crystallographically different materials, we investigate the strain-induced oxygen vacancy stability of charged and neutral vacancies in rocksalt, fluorite, perovskite and pyrochlores based oxides materials using DFT calculations. We show that for a given material there is a window of strain within which an oxygen vacancy is stable; beyond that range, the vacancy can become unstable. Furthermore, we distinguish between chemical expansion of charged and neutral vacancies. The results demonstrate that neutral vacancies lead to lattice expansion; while in contrast, charged vacancies lead to lattice contraction, i.e., they are likely to be more stable under compressive strain. These results could serve as guiding principles in predicting oxygen vacancy stability in strained systems and in the design of vacancy stabilized materials.

\section{Methodology}

We perform DFT calculations using the VASP code. ${ }^{24}$ In particular, the projectoraugmented wave (PAW) method with plane waves up to the energy cutoff of $500 \mathrm{eV}$ is used, and the exchange-correlation energy is evaluated by generalized-gradient approximation (GGA) using the Perdew-Burke-Ernzerhof (PBE) functional. ${ }^{25}$ All relaxations were done until the forces were smaller than $0.01 \mathrm{eV} / \mathrm{A}$. We simulate ten systems, i.e., $\mathrm{CeO}_{2}, \mathrm{MgO}, \mathrm{CaO}, \mathrm{BaO}, \mathrm{CaTiO}_{3}, \mathrm{SrTiO}_{3}, \mathrm{BaTiO}_{3}, \mathrm{La}_{2} \mathrm{Ti}_{2} \mathrm{O}_{7}, \mathrm{La}_{2} \mathrm{Zr}_{2} \mathrm{O}_{7}$ and $\mathrm{La}_{2} \mathrm{Hf}_{2} \mathrm{O}_{7}$. Recent research has established that the strong on-site Columbic interaction needs to be corrected for $\mathrm{CeO}_{2}$. The Hubbard $U$-correction is applied to $\mathrm{Ce} 4 f$ electrons as proposed by Dudarev et al., ${ }^{26}$ using a value of $U_{\text {eff }}=5 \mathrm{eV}$ based on the previous works. ${ }^{27}$ 
For $\mathrm{Ti}$ and $\mathrm{Hf}$, we found that the oxygen vacancy effects on lattice expansion are independent of the $U$-correction; therefore, we have not used the correction for the two atoms. All systems are modeled using their $2 \times 2 \times 2$ supercell. In each of these systems, charged and neutral oxygen vacancy is modeled using the Brillouin zone sampled with a $2 \times 2 \times 2$ Monkhrost-pack mesh $k$-points grid. Spin polarization is taken in account in all calculations. After the tests for plane-wave energy cutoff and k-point sampling, the numerical error is found to be less than $1 \mathrm{meV} / \mathrm{atom}$, illustrating energy convergence for supercell size and k-points. The bulk lattice parameters obtained for all systems are within $2 \%$ of the experimental lattice constants.

\section{Results}

\section{Oxygen vacancy stability in an interface structure:}

We first present an example of oxygen vacancy stability across an interface structure formed between fluorite-based $\mathrm{CeO}_{2}$ and $\mathrm{ZrO}_{2}$. The interface calculations are performed to show that the stability of the oxygen vacancy can be manipulated by applied strain. The supercell contains $1 \times 2 \times 12$ unit cells ( 288 atoms) in total, in which $1 \times 2 \times 4$ unit cell of $\mathrm{ZrO}_{2}$ are sandwiched between $1 \times 2 \times 8$ unit cells of $\mathrm{CeO}_{2}$. Both materials are interfaced using (001) terminations sharing oxygen plane of atoms. The structure is fully relaxed using DFT and the final structure is used as the input structure for oxygen vacancy calculations. An oxygen vacancy is created by removing an oxygen atom from inside $\mathrm{CeO}_{2}$ and from the interface in separate calculations. The oxygen vacancy image distance in the $y$ direction is 2 lattice parameters $(\sim 11.06 \AA)$; whereas in the $x$ direction, it is 1 lattice parameter $(\sim 5.41 \AA)$. While the image distance in $\mathrm{x}$ is relatively small, we tested our calculations by doubling the supercell in the $x$ direction to a total of 576 atoms and 
did not find any significant difference in the relative energy/stability of the oxygen vacancy. Since we plot the oxygen vacancy stability relative to the interface (see Figure 1a), the image interaction is expected to cancel out. (Here, the oxygen vacancy stability is defined as system energy containing oxygen vacancy in $\mathrm{CeO}_{2}$ compared to that at the interface). Due to this reason and the high computational cost associated with the 576 atoms supercell, we chose to perform our simulations on the 288 atoms supercell. After full relaxation, the cell is then equally strained in the $x$ and $y$ directions that form the interfacial plane, and the system energy is calculated as a function of strain. The stability of oxygen vacancy inside $\mathrm{CeO}_{2}$ (shown in square symbols) relative to that at the interface (in diamonds) is shown in Figure 1a. In the unstrained case, the oxygen vacancy is more stable inside $\mathrm{CeO}_{2}$ by $0.25 \mathrm{eV}$. Upon compression, the stability of the vacancy starts to change, and the vacancy is almost equally stable in $\mathrm{CeO}_{2}$ and at the interface. On the tensile side, we see a similar, but gradual effect. Whereas the vacancy is stable in $\mathrm{CeO}_{2}$ at up to $3 \%$ strain, as shown in Figure 1a, the stability gradually decreases with increasing tensile strain. Eventually, at larger tensile strains, the stability will reverse similar to that in the compressed case. These simulations show that oxygen vacancies could be functionalized with the applied strain, and their stabilities can be desirably manipulated. This stability behavior can be understood and to some extent predicted by a simple model using bulk single crystal. Here, we perform single crystal DFT calculations on a $2 \times 2 \times 2$ supercell bulk $\mathrm{CeO}_{2}$, where the supercell is hydrostatically strained and the total energy of the system containing a vacancy is followed. This energy profile (or vacancy stability) relative to the unstrained case is shown in Figure $1 \mathrm{~b}$ and is obtained using equation 1.

$$
E^{s, v}=E^{s, v} \quad E^{o, v}
$$



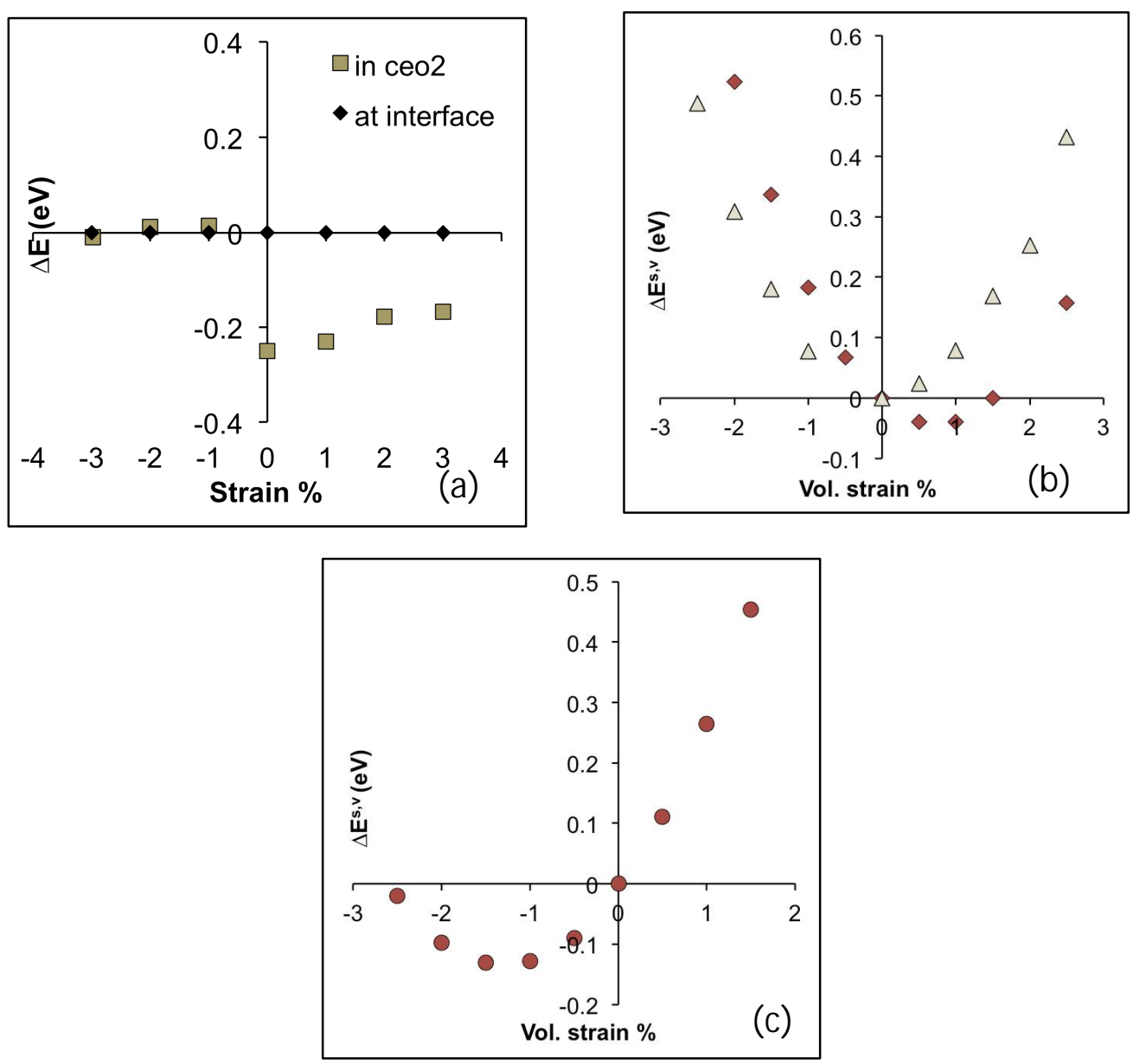

Figure 1. (a) Oxygen vacancy stability in a $\mathrm{CeO}_{2}-\mathrm{ZrO}_{2}$ interface structure as a function of two-dimensional strain. The vacancy is separately placed inside $\mathrm{CeO}_{2}$ and at the interface, and the difference in the total system energy is plotted. Square symbols represent stability of vacancy inside $\mathrm{CeO}_{2}$ with respect to interface (black diamonds). At zero strain, the vacancy is stable inside $\mathrm{CeO}_{2}$. Compression and tensile strain leads to decrease in the stability. (b) Vacancy stability in a $2 \times 2 \times 2 \mathrm{CeO}_{2}$ supercell containing a neutral vacancy (red diamonds). An equation-of-state for the pure system is plotted in hollow triangles. (c) Vacancy stability in a $2 \times 2 \times 2 \mathrm{CeO}_{2}$ supercell containing a +2 charged vacancy.

Here, $\Delta E^{s, v}$ is the change in the system energy containing a single vacancy at a given strain, $s, E^{s, v}$ is the total energy of the strained system, and $E^{o, v}$ is the total energy of the unstrained system. We also plot the energy profile of the system without a vacancy, i.e., equation-of-state as shown by red diamonds. As observed in Figure 1b, the minimum 
energy for a system containing a vacancy is observed not at the equilibrium lattice parameter, i.e., at 0 ; instead it occurs under tensile strain revealing expansion of the lattice by the oxygen vacancy, thus stabilizing the vacancy under larger volume. The depth in the parabola is representative of the volume change caused by the oxygen vacancy. Further tensile strain, however, destabilizes the vacancy as shown by the parabolic nature of the curve demonstrating that the vacancy is stable only within a certain range of tensile strain. This is similar to the trend observed above for the interface structure, where the vacancy is only stable within a certain window of strain, particularly on the tensile side. Similar discussion on other interface structures and oxygen stabilities can be found elsewhere. ${ }^{28}$

The above results are obtained for the neutral vacancy, and these are consistent with previous reports ${ }^{19-23}$ that predicted: (1) the oxygen vacancy possesses a larger volume, which leads to lattice expansion, and (2) it stabilizes under tensile conditions. Now we draw attention to the +2 charge state of the oxygen vacancy that has not been discussed previously in greater detail. In general, the thermodynamic stability of neutral or charged vacancies during technological applications is dependent on the operating temperature and oxygen partial pressure conditions, and their transition from one to the other has been widely investigated in literature. ${ }^{29-32}$ Similar to Figure $1 \mathrm{~b}$, the energy profile for the charged vacancy is provided in Figure 1c and is found to be opposite to that of the neutral vacancy, i.e., the charged vacancy has smaller volume than the oxygen atom, and the lattice undergoes compression. This indicates that the charged vacancy is likely to be more stable under compressed strain rather than tensile strain. These opposite behaviors could have contrasting effects on vacancy concentration profiles in interfacial 
materials and in materials under extreme conditions. In the following sections, we discuss these contrasting effects in different oxide materials.

\section{Vacancy stability in rocksalt, perovskite and pyrochlore structures}

Having illustrated chemical expansion effects in fluorite ceria, we now discuss similar effects in rocksalt, perovskite and pyrochlores structures. For each of these structures, three model materials are chosen with different cations within the same Group of the periodic table for a given structure.

\subsection{Rocksalt}

We choose $\mathrm{MgO}, \mathrm{CaO}$ and $\mathrm{BaO}$ as our three model materials for the rocksalt structure. The lattice parameters obtained from our DFT calculations are 4.24, 4.83 and $5.60 \AA$, respectively. Similar to Figures $1 \mathrm{~b}$ and $1 \mathrm{c}$, the $\Delta E^{s, v}$ profiles for both neutral and charged vacancies for $\mathrm{MgO}, \mathrm{CaO}$ and $\mathrm{BaO}$ are shown in Figures $2 \mathrm{a}, 2 \mathrm{~b}$ and $2 \mathrm{c}$, respectively. In addition, a comparison among the three materials for the neutral and charged vacancies is shown in Figures $2 \mathrm{~d}$ and $2 \mathrm{e}$ respectively.

The results demonstrate that the neutral vacancies are stable under lattice expansion while the charged vacancies are stable under lattice compression, consistent with the above observation in $\mathrm{CeO}_{2}$. By comparing Figures $2 \mathrm{~d}$ and $2 \mathrm{e}$, we find that the relaxation of the vacancies depends on the lattice parameter or the cation radii. For the neutral vacancy, the lattice expansion is largest in $\mathrm{BaO}$, and smallest in $\mathrm{MgO}$. In contrast, for the charged vacancy the expansion is largest in $\mathrm{MgO}$ and smallest in $\mathrm{BaO}$, thus showing opposite trends between the two vacancies. The energy relaxation (or stability), which is measured by the depth of the parabolic curve with respect to the unstrained case, is larger for the charged vacancy as compared to the neutral vacancy. 

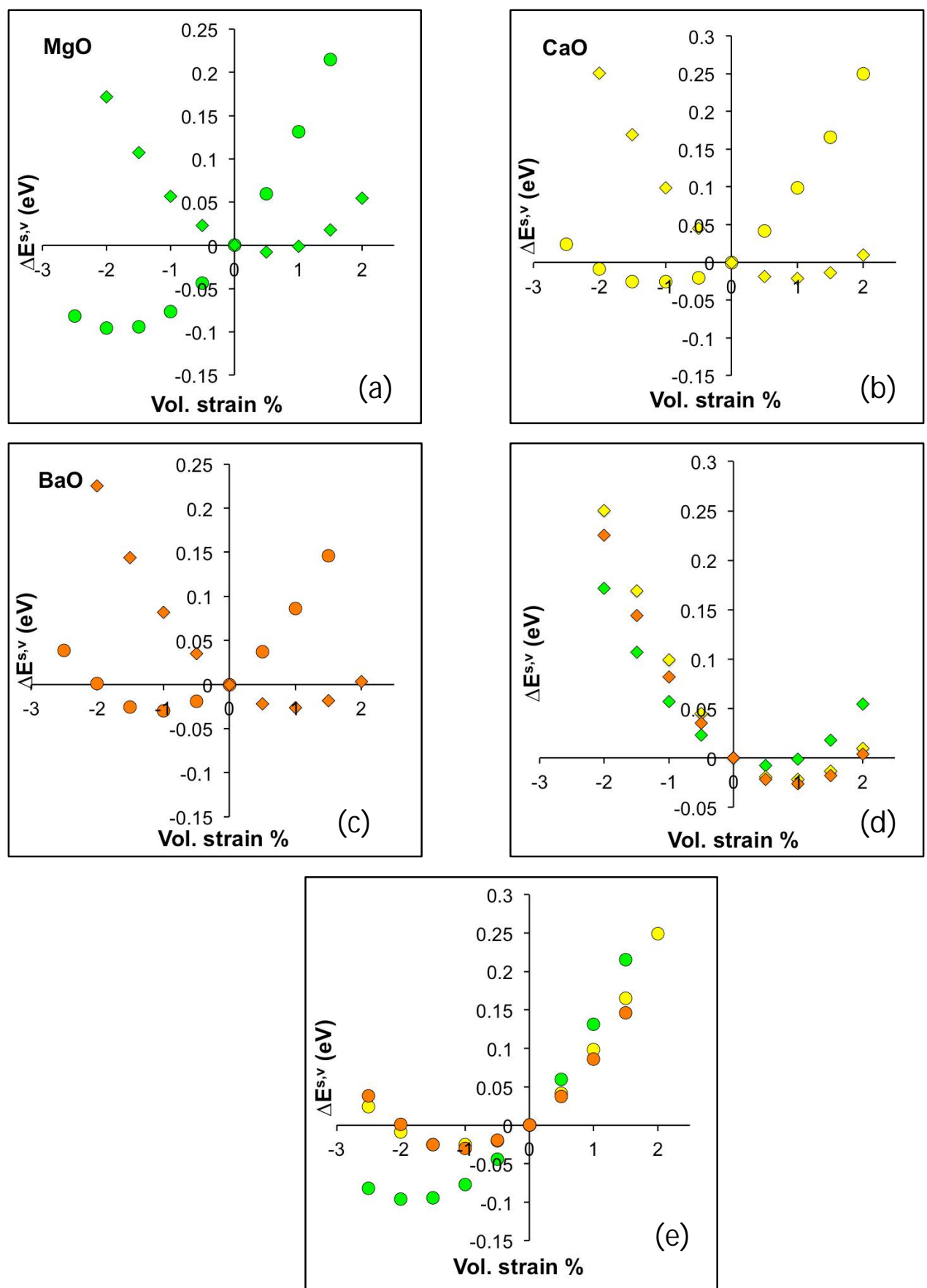

Figure 2. Stability of a neutral (diamonds) and charged oxygen vacancy (circles) in (a) $\mathrm{MgO}$, (b) $\mathrm{CaO}$ and (c) $\mathrm{BaO}$. Comparison of (d) neutral and (e) charged oxygen vacancy among the three rocksalt structures.

The window of vacancy stability in $\mathrm{MgO}$ as a function of strain is larger for the charged vacancy compared to the neutral vacancy - whereas the neutral vacancy is only stable 
within $1 \%$ tensile strain, the charged vacancy is stable for more than $3 \%$ compressive strain. This window difference is however less pronounced in $\mathrm{CaO}$ and $\mathrm{BaO}$. The window of stability could provide a degree of freedom in functionalizing a material. From these results, an overall trend emerges that in rocksalt materials, the stability of charged vacancies under compressed strain decreases by going from top to bottom of the cation Group, whereas it increases for the neutral vacancies.

\subsection{Perovskites}

We choose $\mathrm{CaTiO}_{3}, \mathrm{SrTiO}_{3}$ and $\mathrm{BaTiO}_{3}$ as three model materials for the perovskite structure. While $\mathrm{CaTiO}_{3}$ exists in orthorhombic (Pbnm) structure at room temperature, we model the high temperature cubic phase of the perovskite structure for a consistent analysis with the other two materials. The lattice parameters obtained from the

DFT calculations on bulk structures of CTO, STO and BTO are 3.89, 3.94 and $4.035 \AA$ respectively. Similar to Figure 2, the $\Delta E^{s, v}$ profiles for both neutral and charged vacancies for CTO, STO and BTO are shown in Figures $3 \mathrm{a}, 3 \mathrm{~b}$ and $3 \mathrm{c}$ respectively. ${ }^{33}$ The comparison among the three materials for the neutral and charged vacancies is also shown in Figures $3 \mathrm{~d}$ and $3 \mathrm{e}$ respectively.

We find that the perovskite structures also show similar behavior for both charged and neutral vacancies as above, i.e., neutral vacancies are stable under lattice expansion, and charged vacancies are stable under lattice compression. From Figure $2 \mathrm{~d}$ for the neutral vacancies, the lattice expansion is largest in $\mathrm{BaTiO}_{3}$ and smallest in $\mathrm{CaTiO}_{3}$; whereas for charged vacancies, the expansion is largest in $\mathrm{CaTiO}_{3}$ and smallest in $\mathrm{BaTiO}_{3}$ showing similar cation-radii trend as above in the rocksalt structure. For all the three 
materials, the energy relaxation (or stability) of the charged vacancy is larger than for the neutral vacancy, in agreement with the trend in the rocksalt structure.
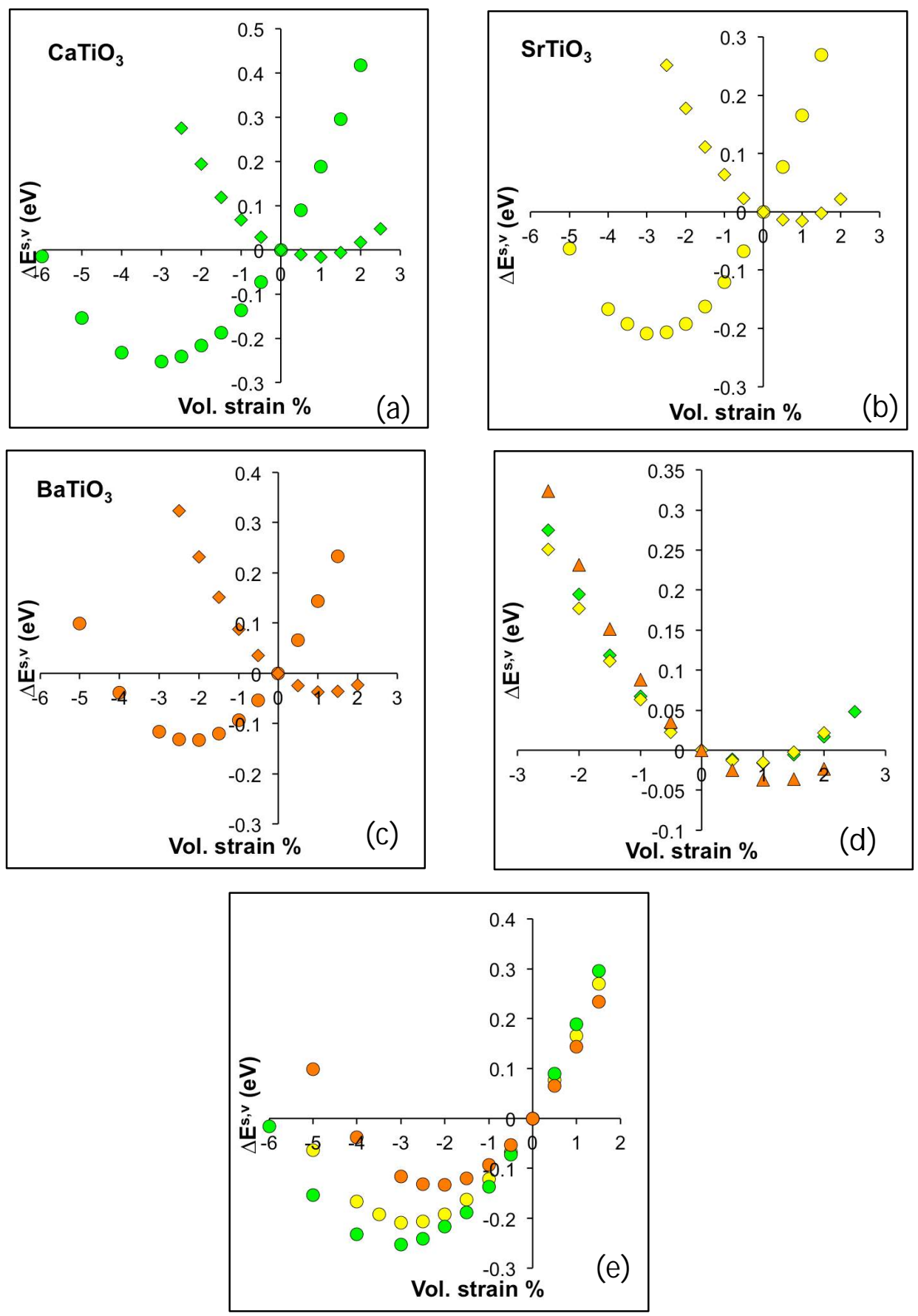

Figure 3. Stability of a neutral (diamonds) and charged oxygen vacancy (circles) in (a) $\mathrm{CaTiO}_{3}$, (b) $\mathrm{SrTiO}_{3}$ and (c) $\mathrm{BaTiO}_{3}$. Comparison of (d) neutral and (e) charged oxygen vacancy among the three perovskite structures. 
The window of vacancy stability trend is also consistent with the rocksalt structure; for the charged vacancy, the smallest cation perovskite, $\mathrm{CaTiO}_{3}$, has the largest window; whereas it is smallest for $\mathrm{BaTiO}_{3}$. In addition, we find that perovskites have a large window (of up to $\sim 6 \%$ vol. strain for $\mathrm{CaTiO}_{3}$ ), indicating that it may be possible to gain large control over their vacancy functionalization.

\subsection{Pyrochlores}

We choose La-based pyrochlores and vary the B cations (from Group IVB) in this $\mathrm{A}_{2} \mathrm{~B}_{2} \mathrm{O}_{7}$ structure. The materials discussed here are $\mathrm{La}_{2} \mathrm{Ti}_{2} \mathrm{O}_{7}, \mathrm{La}_{2} \mathrm{Zr}_{2} \mathrm{O}_{7}$ and $\mathrm{La}_{2} \mathrm{Hf}_{2} \mathrm{O}_{7}$. The lattice parameters obtained for LTO, LZP and LHO are 10.485, 10.854 and $10.802 \AA$ respectively. In the pyrochlore structure, there are two types of oxygen atoms, crystallographically characterized as $\mathrm{O}-8 \mathrm{~b}$ and $\mathrm{O}-48 \mathrm{f}$, and distinguished by their coordination to cations. The oxygen $\mathrm{O}-8 \mathrm{~b}$ atoms are tetrahedrally coordinated only to Atype cations, whereas the oxygen $\mathrm{O}-48 \mathrm{f}$ atoms are tetrahedrally coordinated to two A and two B cations. Due to this difference in coordination, the two oxygen atoms have different bond lengths. In the following analysis, we discuss the stability behavior of both of them.

The energy profile of neutral O-8b and O-48f vacancies are shown in Figures 4a, $4 \mathrm{~b}$ and $4 \mathrm{c}$. The data for the $\mathrm{O}-8 \mathrm{~b}$ vacancy is shown in grey diamonds, and that for $\mathrm{O}-48 \mathrm{f}$ is shown in black diamonds. It is found that the stability profile for the $\mathrm{O}-8 \mathrm{~b}$ vacancy in all three pyrochlores is different from the previous structures, i.e., here, the minimum in the parabola that corresponds to lattice expansion is not observed under tensile strain. Instead it lies almost at 0 , corresponding to the equilibrium lattice parameter. This shows that the $\mathrm{O}-8 \mathrm{~b}$ vacancy may not have significant chemical expansion, and could be 
expected to be stable with the bulk equilibrium lattice constant. In contrast, the O-48f vacancy is stable under $1 \%$ volume strain in $\mathrm{La}_{2} \mathrm{Ti}_{2} \mathrm{O}_{7}$. However, as we go down the Group by replacing $\mathrm{Ti}$ with $\mathrm{Zr}$ and $\mathrm{Hf}$, this stability decreases under tensile strain, and the O-48f vacancy behaves very similar to the $\mathrm{O}-8 \mathrm{~b}$ vacancy. These results show that the O$8 \mathrm{~b}$ vacancy may not actively respond to tensile strain for stabilization and that the ability to stabilize the $\mathrm{O}-48 \mathrm{f}$ vacancy is reduced in $\mathrm{La}_{2} \mathrm{Zr}_{2} \mathrm{O}_{7}$ and $\mathrm{La}_{2} \mathrm{Hf}_{2} \mathrm{O}_{7}$ compared to $\mathrm{La}_{2} \mathrm{Ti}_{2} \mathrm{O}_{7}$. On a broader view, due to the presence of two different oxygen vacancies in the pyrochlores, there is a possibility to desirably functionalize only one oxygen atom (O48f) out of the two, thus providing a degree of freedom in $\mathrm{La}_{2} \mathrm{Ti}_{2} \mathrm{O}_{7}$ (as also observed in $\mathrm{CaMnO}_{3}$ previously); however, this control appears to diminish in $\mathrm{La}_{2} \mathrm{Zr}_{2} \mathrm{O}_{7}$ and $\mathrm{La}_{2} \mathrm{Hf}_{2} \mathrm{O}_{7}$.

The oxygen stability profile of the charged vacancies for the same three systems is shown in Figures $4 \mathrm{~d}, 4 \mathrm{e}$ and $4 \mathrm{f}$. In contrast to the anomalous behavior of the $\mathrm{O}-8 \mathrm{~b}$ vacancy under tensile strain, here we find that it is stable under compressive strain, thus following the normal trend as observed in the other materials above. The stability of the $\mathrm{O}-8 \mathrm{~b}$ vacancy is also more pronounced than the $\mathrm{O}-48 \mathrm{f}$ vacancy in all three materials, as shown by the relative depths of the parabolic curves. In addition, the stability window is also larger for the $\mathrm{O}-8 \mathrm{~b}$ vacancy than for the $\mathrm{O}-48 \mathrm{f}$ vacancy in all three materials. A comparison of the stability profiles for the $\mathrm{O}-8 \mathrm{~b}$ vacancy among the three materials is shown in Figure 4g. Again we find that there is a trend for the chemical expansion and stability among the three materials. Similar to rocksalt and pyrochlores, the chemical expansion and stability are largest in the smallest B-type cation, i.e., Ti, and it decreases going down the Group. 

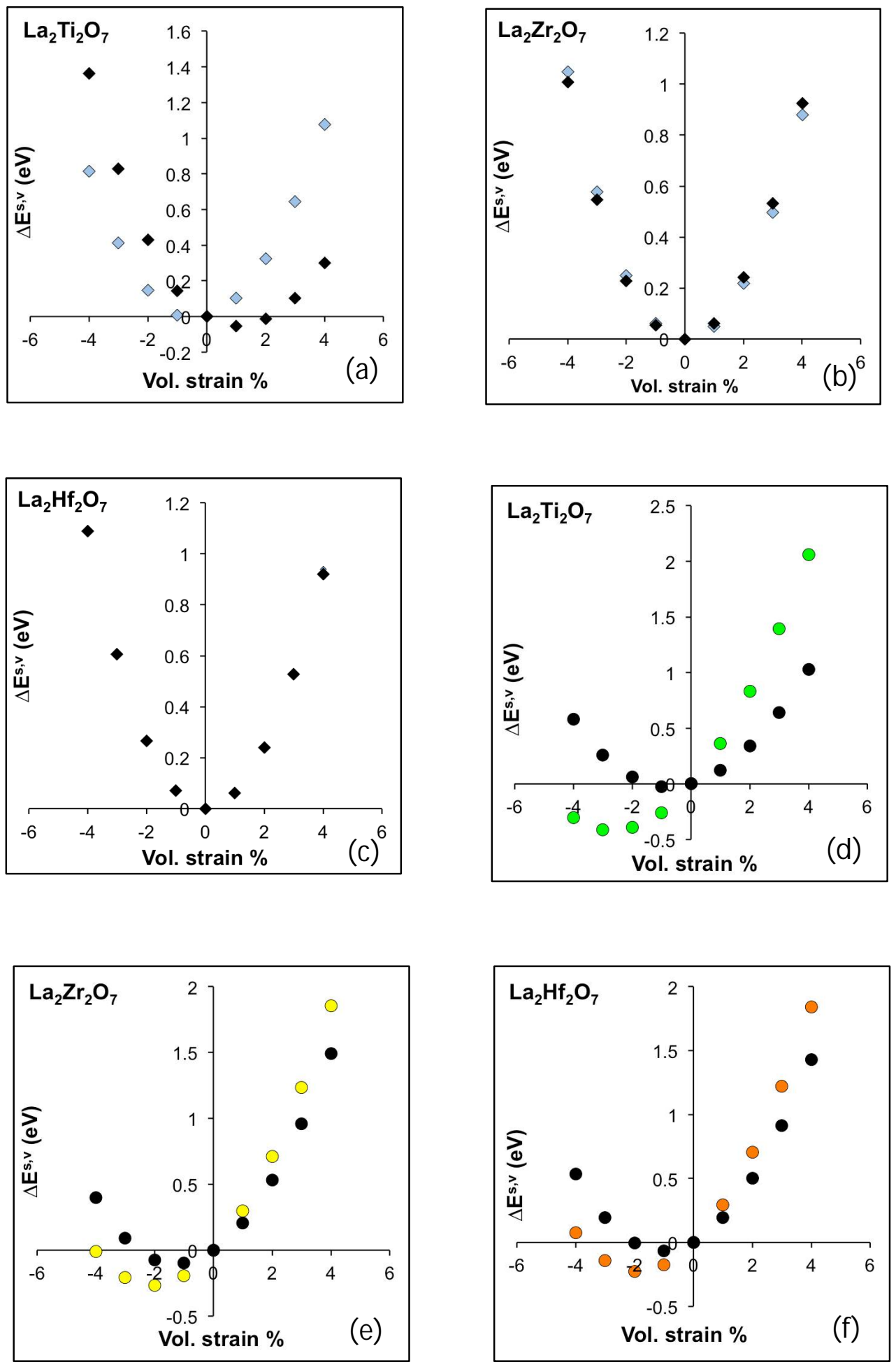


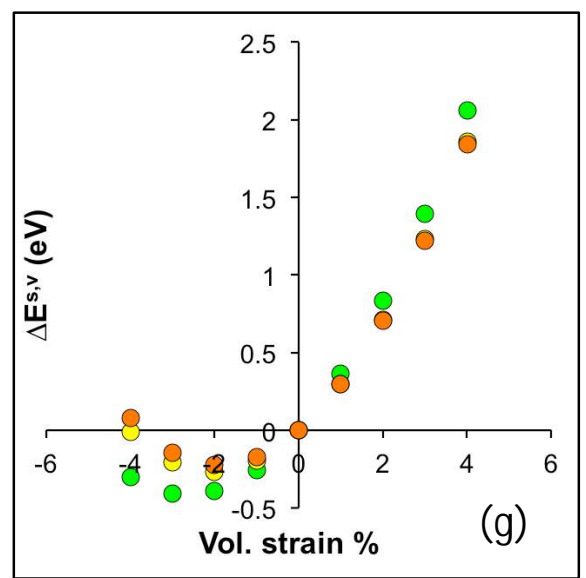

Figure 4. Comparison of neutral oxygen vacancy $\mathrm{O}-8 \mathrm{~b}$ (grey diamonds) and O-48f (black diamonds) stability in (a) $\mathrm{La}_{2} \mathrm{Ti}_{2} \mathrm{O}_{7}$, (b) $\mathrm{La}_{2} \mathrm{Zr}_{2} \mathrm{O}_{7}$, and (c) $\mathrm{La}_{2} \mathrm{Hf}_{2} \mathrm{O}_{7}$. Similar comparison for charged oxygen vacancy O-8b (colored circles) and O-48f (black circles) stability in (d) $\mathrm{La}_{2} \mathrm{Ti}_{2} \mathrm{O}_{7}$, (e) $\mathrm{La}_{2} \mathrm{Zr}_{2} \mathrm{O}_{7}$, and (f) $\mathrm{La}_{2} \mathrm{Hf}_{2} \mathrm{O}_{7}$. (g) Comparison of charged oxygen vacancy $\mathrm{O}-8 \mathrm{~b}$ among three materials.

\section{Conclusions}

A fundamental concept on gaining control over kinetic and thermodynamic defect properties via strain is emerging. On the kinetics front, diverse studies have now largely shown that oxygen vacancy migration barriers can be significantly lowered via tensile strain. However, there is still a large quantitative discrepancy, particularly on the wide gap between the conductivity results among different groups. ${ }^{11,34,35}$ This gap showcases our limited understanding on the structural issues of interfaces, and limited control over point defects. On the thermodynamic front, this strained-induced defect stability understanding is even less developed. Recent reports, both from experiments and computation, ${ }^{19-22,}{ }^{28,}$ Error! Hyperlink reference not valid.' Error! Hyperlink reference not valid. have indicated that strain could be used to manipulate defect concentrations. However, as shown in this work, the degree of control may largely 
depend on the material and the crystal structure. Therefore, studies that characterize such effects in relevant crystal structures and different chemical compositions, such as by Marrocchelli et al. ${ }^{22}$ on fluorite materials, are required to gain control over chemical expansion defect behaviors.

The results obtained from bulk calculations in this work indicate that charged and neutral vacancies may respond very differently to strain. In the case of layered materials, particularly for ion-transport applications, the tensile strain would have an additive effect on the neutral vacancy diffusivity, i.e., not only do the migration barriers decrease with strain, but the concentration of neutral vacancies also increases. In contrast, the effect on charged vacancies may not be additive. From the above results, the tensile strain seems to lower their concentration, thus decreasing their contribution to conductivity. However, in addition to these bulk calculations, a simultaneous understanding of oxygen vacancy stability in interface systems is also important where the relative stability could change depending upon the amount of strain applied. In a recent work, we showed that oxygen vacancy could be stabilized on either side of the interface by manipulating interfacial strain thus necessitating a need for much deeper understanding of oxygen vacancy thermodynamics in strained interfacial materials. ${ }^{28}$ Similarly, the tensile-strain effect would need to be studied separately for inequivalent oxygen atoms within a given material that have different coordination environment, as shown by the two different oxygen sites in the pyrochlores. As observed above in $\mathrm{La}_{2} \mathrm{Ti}_{2} \mathrm{O}_{7}$ or previously in $\mathrm{CaMnO}_{3},{ }^{23}$ the different oxygen sites present an opportunity to favorably functionalize them separately, thus opening another degree of freedom. Similarly, nuclear materials that are strained due to temperature and chemical gradients may have significant strain 
contribution to the formation of defect complexes, such as voids and dislocation loops. Both neutral and charged oxygen vacancies are present in materials under irradiation, and their underlying effects on grain growth have been recently observed in zirconia and ceria. $^{38-40}$ However, the key mechanisms to unintuitive grain growth under cryogenic temperatures, attributed to charged vacancies, are being further explored. Similar effects on phase separation in nuclear fuels and pyrochlore materials for waste-storage may be important.

In conclusion, this work on separating the chemical expansion of charged and neutral vacancies on fluorite, rocksalt, perovskite and pyrochlore materials shows that the two vacancies behave oppositely under strain. Whereas the neutral vacancy leads to lattice expansion, the charged vacancy leads to compression. This observation highlights their behavior under strain, i.e., the neutral and charged vacancies are expected to be stable under tensile strain and compressive strain respectively.

Acknowledgement: This work was supported by the U.S. Department of Energy, Office of Science, Basic Energy Sciences, Materials Science and Engineering Division. The computer simulations on fluorite structures were carried out using the resources of the National Energy Research Scientific Computing Center, which is supported by the Office of Science, U.S. Department of Energy under Contract No.DEAC02-05CH11231. The computer simulations on the other structures were carried out using the Cascade computer cluster at the Environmental Molecular Sciences Laboratory (EMSL), a national scientific used facility sponsored by United States Department of Energy, located at Pacific Northwest National Laboratory. 


\section{References}

1. J. Maier, Nature Materials, 2005, 4, 805-815.

2. H. L. Tuller and S. R. Bishop, Annual Review of Materials Research, 2011, 41, 369-398.

3. E. D. Wachsman and K. T. Lee, Science, 2011, 334, 935-939.

4. R. Waser, R. Dittmann, G. Staikov and K. Szot, Advanced Materials, 2009, 21, 2632-2663.

5. M.-J. Lee, C. B. Lee, D. Lee, S. R. Lee, M. Chang, J. H. Hur, Y.-B. Kim, C.-J. Kim, D. H. Seo, S. Seo, U.-I. Chung, I.-K. Yoo and K. Kim, Nature Materials, 2011, 10, 625-630.

6. S. V. Kalinin and N. A. Spaldin, Science, 2013, 341, 858-859.

7. Y.-M. Kim, J. He., M. D. Beigalski, H. Ambaye, V. Lauter, H. M. Christen, S. T. Pantelides, S. J. Pennycook, S. V. Kalinin and A. Y. Borisevich, Nature Materials, 2012, 11, 888-894.

8. $\quad$ A. Ourmazd and J. C. H. Spence, Nature (London), 1987, 329, 425-427.

9. $\quad$ R. Devanathan and W. J. Weber, Journal of Materials Research, 2011, 23, 593597.

10. D. G. Schlom, L.-Q. Chen, C. J. Fennie, V. Gopalan, D. A. Muller, X. Pan, R. Ramesh and R. Uecker, MRS Bulletin, 2014, 39, 118-130.

11. B. Yildiz, MRS Bulletin, 2014, 39, 147-156.

12. I. J. Beyerlein, A. Caro, M. J. Demkowicz, N. A. Mara, A. Misra and B. P. Uberuaga, Materials Today, 2013, 16, 443-449.

13. W. Han, M. J. Demkowicz, N. A. Mara, E. Fu, S. Sinha, A. D. Rollett, Y. Wang, J. S. Carpenter, I. J. Beyerlein and A. Misra, Advanced Materials, 2013, 25, 69756979.

14. A. Ohtomo and H. Y. Hwang, Nature, 2004, 427, 423-426.

15. R. Ramesh, Current Science, 2013, 105, 1107-1114.

16. D. S. Aidhy, Y. Zhang and W. J. Weber, ACS Applied Materials and Interfaces, 2014, 6, 15536 - 15541.

17. J. Garcia-Barriocanal, A. Rivera-Calzada, M. Varela, Z. Sefrioui, E. Iborra, C. Leon, S. J. Pennycook and J. Santamaria, Science, 2008, 321, 676-680.

18. N. Schichtel, C. Korte, D. Hesse and J. Janek, Physical Chemistry Chemical Physics, 2009, 11, 3043-3048.

19. D. Marrocchelli, S. R. Bishop, H. L. Tuller, G. W. Watson and B. Yildiz, Physical Chemistry Chemical Physics 2012, 14, 12070-12074.

20. D. Marrocchelli, S. R. Bishop, H. L. Tuller and B. Yildiz, Advanced Functional Materials, 2012, 22, 1958-1965.

21. S. R. Bishop, D. Marrocchelli, W. Fang, K. Amezawa, K. Yashiro and G. W. Watson, Energy \& Environmental Science, 2013, 6, 1142-1146.

22. D. Marrocchelli, S. R. Bishop and J. Kilner, Journal of Materials Chemistry A, 2013, 1, 7673-7680.

23. U. Aschauer, R. Pfenninger, S. M. Selbach, T. Grande and N. A. Spaldin, Phys Rev B, 2013, 88, 054111 (1)-(7).

24. G. Kresse and J. Furthmuller, Computational Materials Science, 1996, 6, 15-50. 
25. J. P. Perdew, K. Burke and M. Ernzerhof, Physical Review Letters, 1996, 77, 3865-3868.

26. S. L. Dudarev, G. A. Botton, S. Y. Savrasov, C. J. Humphreys and A. P. Sutton, Phys Rev B, 1998, 57, 1505-1509.

27. H. Y. Xiao and W. J. Weber, The Journal of Physical Chemistry. B, 2011, 115, 6524-6533.

28. D. S. Aidhy, B. Liu, Y. Zhang and W. J. Weber, The Journal of Physical Chemistry B, DOI: $10.1021 / \mathrm{jp} 507876 \mathrm{~m}$.

29. A. Samanta, W. E and S. B. Zhang, Phys Rev B, 2012, 86, 195107 (1)-(14).

30. F. Oba, M. Choi, A. Togo and I. Tanaka, Science and Technology of Advanced Materials, 2011, 12, 034302 (1)-(14).

31. H. Xu, D. Lee, J. He, S. Sinnott, V. Gopalan, V. Dierolf and S. Phillpot, Phys Rev $B, 2008,78,174103$ (1) - (12).

32. J. Zheng, G. Ceder, T. Maxisch, W. Chim and W. Choi, Phys Rev B, 2007, 75, 104112 (1) - (7).

33. While larger system sizes are generally required for defect calculations, our choice of $2 \times 2 \times 2$ supercell is guided by the volume change that we intend to capture which is rather a local phenomenon. In addition, since we are not calculating vacancy formation energy but rather the relative energy change, the image interactions are expected to cancel out.

34. D. S. Aidhy, Y. Zhang and W. J. Weber, The Journal of Physical Chemistry C, 2014, 118, 4207-4212.

35. D. S. Aidhy, Y. Zhang and W. J. Weber, Journal of Materials Chemistry A, 2014, 2, 1704-1709.

36. S. Estradé, J. Arbiol, F. Peiró, L. Abad, V. Laukhin, L. Balcells and B. Martínez, Applied Physics Letters, 2007, 91, 252503 (1)-(3).

37. S. Estradé, J. Arbiol, F. Peiró, I. C. Infante, F. Sánchez, J. Fontcuberta, F. de la Peña, M. Walls and C. Colliex, Applied Physics Letters, 2008, 93, 112505 (1)-(3).

38. Y. Zhang, W. Jiang, C. Wang, F. Namavar, P. D. Edmondson, Z. Zhu, F. Gao, J. Lian and W. J. Weber, Phys Rev B, 2010, 82, 184105 (1)-(7).

39. D. S. Aidhy, Y. Zhang and W. J. Weber, Physical Chemistry Chemical Physics, 2013, 15, 18915-18920.

40. Y. Zhang, D. S. Aidhy, T. Varga, S. Moll, P. D. Edmondson, F. Namavar, K. Jin, C. N. Ostrouchov and W. J. Weber, Physical Chemistry Chemical Physics, 2014, 16, 8051-8059. 


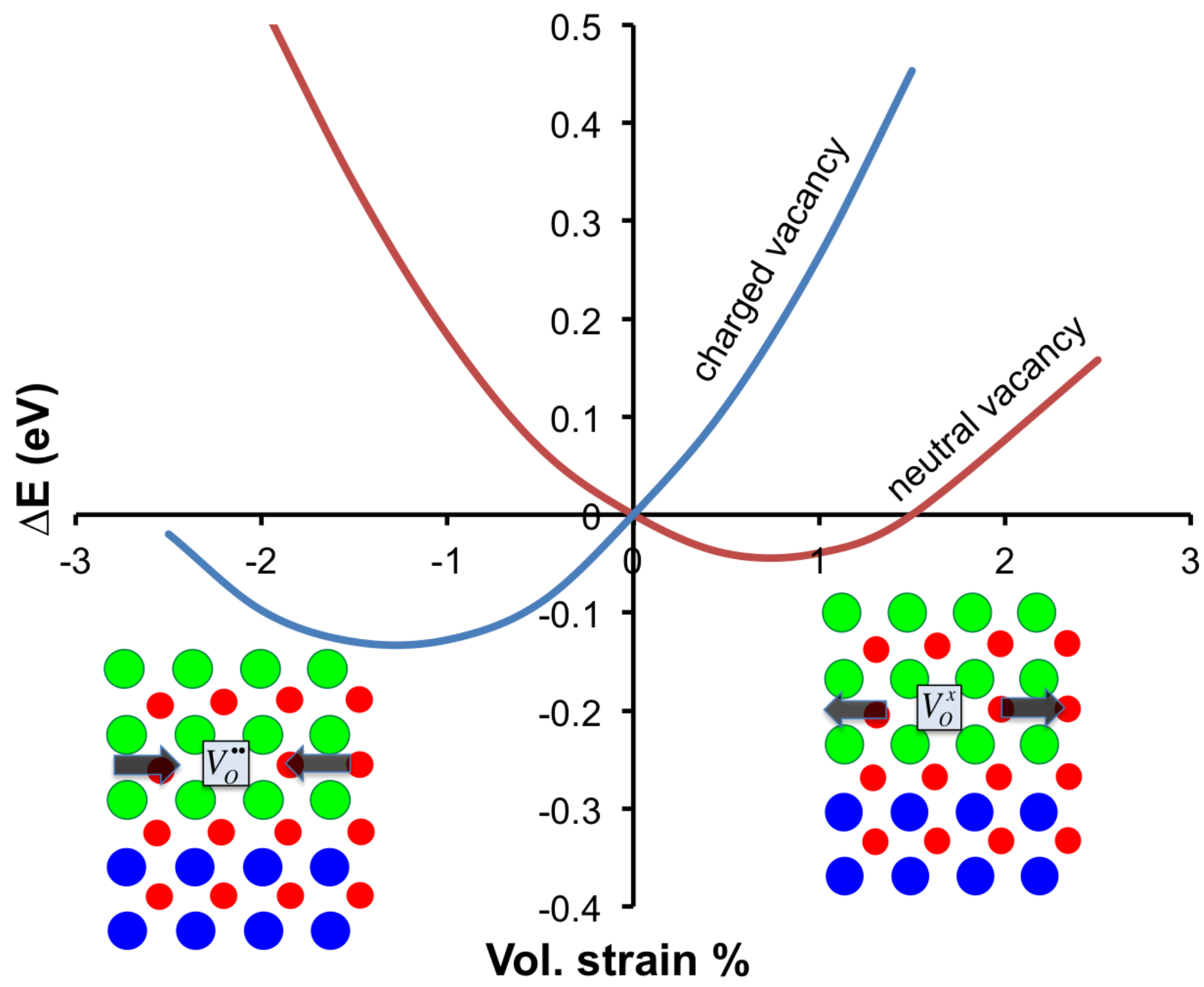

